\title{
A GIS Model for Analyzing Airspace Obstructions and Safety near Airports
}

\author{
Sze-Wei Chang \\ Department of Aviation Management, China University of Science and Technology, Hsinchu 31241, Taiwan
}

\begin{abstract}
The purpose of Federal Aviation Regulations Part 77, entitled "Objects Affecting Navigable Airspace", is to identify potential hazards and prevent adverse impacts to the safe and efficient use of navigable airspace. The OIS (obstruction identification surfaces) represent these regulations, identify objects that penetrate these imaginary surfaces, evaluate hazardous effects, and ensure safe separations of aircraft from obstructions. The OIS evaluation is often used for selecting airfield locations and runway orientations. More importantly, the OIS evaluation is an airport's safeguard against flawed urban development, especially for airports near rapidly expanding cities. Without accurate analysis tools, hand-calculation errors on the OIS can occur and cause adverse impacts to airport safety. Applying the GIS (geographic information system) features of geospatial data and 3D formats, this paper proposes a model named GIS Obstruction Model which is capable of precisely calculating maximum allowable heights and creating visual imagery of penetrating obstructions. The special features of the model include web-based design, adaptability to any airport, user-friendly multiple inquiry methods, quick response to online inquiries, and response with 3D visual reality. The proposed GIS Obstruction Model is useful for airport planning, architecture design, safety management or civil engineering permit review.
\end{abstract}

Key words: Obstruction identification surfaces, geographic information systems, airport design, obstruction evaluation, obstacle limitation surfaces.

\section{Introduction}

Airspace protection and obstacle clearance are vital to airport and aircraft operation. Restrictions should be established on the heights of buildings, antennas, trees and other objects as necessary to protect the airspace needed for safe operation of the airport and aircraft. The methods for determining the complex airport imaginary surfaces are the regulations of the FAR (Federal Aviation Regulations) Part 77 "Objects Affecting Navigable Airspace" [1]. They are used to identify potential aeronautical hazards, thus preventing or minimizing adverse impacts to the safe and efficient use of navigable airspace.

The OIS (obstruction identification surfaces), which graphically represent the FAR Part 77 regulations, are used to identify objects that penetrate these imaginary surfaces, to evaluate hazardous effects and to ensure

Corresponding author: Sze-Wei Chang, assistant professor, research fields: airport design, airline management, air traffic control and fleet planning. the safe separations between aircraft and obstructions. The OIS evaluation is often used for new airfield location selection and runway orientation determination. More importantly, the OIS evaluation is an airport's safeguard against flawed urban development, especially for airports near rapidly expanding cities. Market forces often lead real estate development interests to propose buildings in the vicinity of an airport. Building developers, of course, are interested in maximizing their return on investment, which is most often achieved by developing to the "highest and best use" potential of their land, and as such can desire to build structures of substantial height above the ground. The proposed building permit evaluation procedures are mainly circulated among local government, airport authority and aviation administration [2]. Without accurate and handy tools, hand-calculation errors on the restrictive height/area of the OIS could occur and caused adverse impacts to airport safety. For example, the Taipei Songshan 
Airport (IATA (International Air Transport Association) airport code: TSA; ICAO (International Civil Aviation Organization) airport code: RCSS) is adversely affected by the obstructing Taipei 101 Building, because mistakes in restrictive area calculations were made when the proposal was reviewed by the Taipei City Government and Civil Aviation Authority [3]. The objective of this paper is to develop a model, called the GIS (geographic information system) Obstruction Model, with the special features of precisely calculating restrictive height/area of the OIS and creating visual imagery of obstacles penetrating them. The model applies a GIS's features of geospatial data and 3D visualization to achieve the overall objective.

\section{Literature Review and Methodology}

Litsheim and Xiao [4] commented that airspace protection and obstacle clearance are vital to airport and aircraft operations. Restrictions should be established on the heights of buildings, antennas, trees, and other objects as necessary to protect the airspace needed for operation of the airports and aircraft. The most commonly used criteria for determining complicated airport obstacles are FAR Part 77 imaginary surfaces. Horonjeff's textbook Planning \& Design of Airports, which is widely-used in colleges and graduate schools for airport related courses, demonstrates many OIS example problems. All of these problems require detailed calculation to firstly determine which surface affects any considered obstruction and then determine the restrictive (i.e., maximum allowable) height of the obstacle. The calculation process is quite complicated and time-consuming. A slight error in obstacle location can result in large errors in the restrictive height [5].

The NOAA (National Oceanic and Atmospheric Administration) have provided aeronautical survey data to the FAA (Federal Aviation Administration) for nearly 60 years. These aeronautical survey data help ensuring the safety of the national airspace system and provide accurate location information for features and obstructions on and near airports. The emergence of GPS (global position system)-based navigation has increased the demand for accurate aeronautical surveyed data. NOAA and FAA are currently developing a new approach to aeronautical surveying in response to this need [6].

Panayotov and Georgiev [7] present a spatial model of the airport navigation space with coarsening from the smaller cells to the larger cells. The model is designed as an analytic tool with the geometrical data in the ESRI (Environmental Systems Research Institute) arc GIS environment. The model uses the concept of octree to represent the airport space as a cube. An octree is a tree data structure in which each parental cube has exactly eight children sub-cubes. Octrees are most often used to partition a three-dimensional space by recursively subdividing it into eight octants. The possible obstruction objects are represented with 3D octrees that are located and analyzed for a conflict with the airport space. Panayotov and Georgiev's paper shows the capability to compare the obstruction and restrictive height, no evidence of visualization of the obstructions that penetrate imaginary surfaces.

Panayotov et al. [8] propose an improved spatial model which a CADD (computer-aided design and drafting)/BIM (building information modeling) module for manmade constructions is added to the previous arc GIS (for airspace runway analysis) and octrees (for scalable 3D presentation of the whole airport space) models. The methods are used in Denver International Airport Planning Office only, not web-based design.

Ulubay and Altan [9] present an overview of spatial data integration from different aspects and explore the role of visualization. They also examine the use of analytical and visual types of derived information in solving spatial problems by presenting a study related to the construction of obstacle-free zones for airports. OLS (obstacle limitation surfaces), the topography and man-made objects were used as cartographic input 
layers in determining dangerous objects for aircraft and final analyses results were presented with $3 \mathrm{D}$ screen displays. In the paper, they mainly examine the ICAO Annex 14 regulations "Obstacle Restriction and Removal" and OLS, which is slightly different from FAR Part 77 "Objects Affecting Navigable Airspace" and OIS [10]. Although discussing the spatial data integration methodologies, this paper is only contented with exploring a framework for integration of complex geographic datasets.

The GIS Obstruction Model presented in this paper is built in a GIS environment. A GIS is a special type of information system concerned with geographically referenced data. A GIS has two distinguishing characteristics that make it different from a standard information system. First, the data in a GIS are spatially referenced with $x-y-z$ or latitude-longitudeelevation 3D coordinates. Second, a GIS has mapping capabilities associated with those coordinates. The GIS Obstruction Model presented here is developed with the Skyline software system. The Skyline can provide user roaming of $3 \mathrm{D}$ terrain and image, modeling of objects near the airport and answering of 3D geo-information inquiring about proposed construction objects. It allows building multiple layers of imaginary surfaces with geo-referenced data by using points, lines, polygons and circles. These imaginary surfaces can be further digitally mapping to the geo-referenced stereo imagery in the vicinity of the airport. Its powerful network feature and terrain server technology efficiently stream massive amounts of 3D geographic and geo-referenced data to thousands of concurrent web-site users, giving each user uninterrupted viewing, roaming, inquiring and answering service. Table 1 shows the comparison of proposed GIS Obstruction Model and previous works.

By applying the analytical and geometric method, the proposed runway endpoints and critical points of the imaginary surfaces along 3D coordinates are constructed according to the FAR Part 77 regulations for specific category of runway. The model is transformed from flat surface coordinates into UTM (universal transverse mercator) coordinate system, and then the WGS84 coordinate system with specific elevation. The transparent imaginary surfaces with different colors, which graphically represent the FAR Part 77 regulations, are generated and overlapped with the geo-referenced stereo imagery in the vicinity of the airport.

\section{FAR Part 77 Objects Affecting Navigable Airspace}

FAR Part 77 establishes standards for determining obstructions to air navigation. The standards apply to existing and constructed objects, trees and terrain. The obstruction identification surfaces, depicting the standards, are used to ensure safe separations between aircraft and obstructions. The dimensions of the imaginary surfaces are based on the category of each runway (e.g., utility runway or runways larger than utility) and the type of approach planned for that runway (e.g., visual, non-precision instrument or precision instrument).

Fig. 1 shows the dimensions of FAR Part 77 imaginary surfaces for various types of runway and approach [1]. As an example, the Category E, most

Table 1 Comparison of the proposed GIS Obstruction Model and previous works.

\begin{tabular}{|l|l|l|l|l|l|}
\hline & Methodology & GIS software & $\begin{array}{l}\text { Regulations } \\
\text { applied for }\end{array}$ & $\begin{array}{l}\text { Max allowable height } \\
\text { online inquiry }\end{array}$ & $\begin{array}{l}\text { 3D visualization/ } \\
\text { obstructions penetration }\end{array}$ \\
\hline Panayotov 2008 [7] & Octrees & ESRI arc & FAA OIS & & Yes/no \\
\hline Panayotov 2012 [8] & $\begin{array}{l}\text { Octrees \& } \\
\text { CADD/BIM }\end{array}$ & ESRI arc & FAA OIS & & Yes/no \\
\hline Ulubay 2002 [9] & $\begin{array}{l}\text { Analytical and } \\
\text { visual }\end{array}$ & Unknown & ICAO OLS & & Yes/yes \\
\hline Proposed GIS model & $\begin{array}{l}\text { Analytical \& } \\
\text { geometric }\end{array}$ & Skyline & FAA OIS & Yes & \\
\hline
\end{tabular}




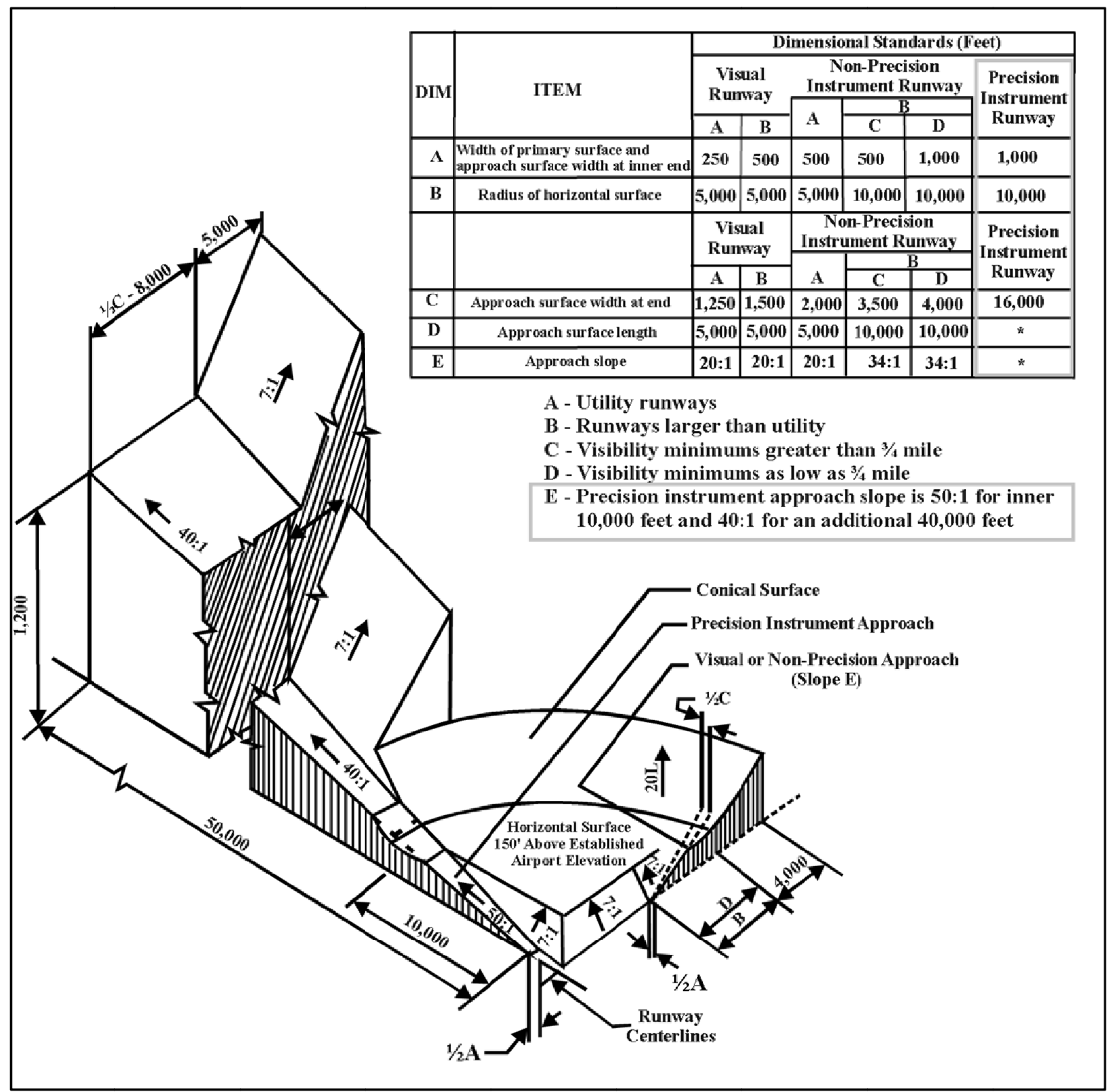

Fig. 1 FAR Part 77 imaginary surfaces [1].

critical OIS for the precision instrument runway with visibility minimums as low as 3/4 miles, is described as follows:

- Primary surface: extends $500 \mathrm{ft}$ on each side of the runway centerline and extends $200 \mathrm{ft}$ beyond each end of the runway;

- Horizontal surface: constructed by swinging arcs of $10,000 \mathrm{ft}$ radii from each end of the primary surface and connecting each arc by tangent lines, with $150 \mathrm{ft}$ above the established airport elevation;
- Conical surface: extends outward and upward from the horizontal surface at a slope of 20 horizontal to 1 vertical for a distance of 4,000 ft;

- Approach surface: extends outward and upward, diverging from the inner width of $1,000 \mathrm{ft}$ to outer end width of $16,000 \mathrm{ft}$, at slopes of 50:1 for the first $10,000 \mathrm{ft}$ of horizontal distance (nearest the runway) and then $40: 1$ for the next $40,000 \mathrm{ft}$ of horizontal distance;

- Transitional surface: extends outward and 
upward at a slope of 7:1 from the primary surface up to the $150 \mathrm{ft}$ horizontal surface, and from the approach surface over a horizontal distance of 5,000 ft.

\section{Design and Features of the Model}

Historically, airport obstruction identification was done with conventional land surveying methods. Although these methods are the most accurate, they are also the most costly and time-consuming. Some GIS obstruction models have been developed in past 10 years. The previous researches create imaginary surface models based on the TIN (triangulated irregular network) technique, instead of full DEM (digital elevation model). The data input of TIN is flexible and fewer points need to be stored than raster DEM. A TIN may be less suited than a raster DEM for certain kinds of GIS applications, such as analysis of a surface's slope and aspect. Based on these reasons, Panayotov et al. [7, 8] propose octree model for scalable 3D presentation of the whole airport space. The octree model is lack of features of transparent imaginary surface overlapping with terrain and, consequently, unable to visually identify the penetrated obstacles.

In this paper, the proposed model generates the multiple layers of imaginary surfaces, which represent the obstruction regulations, with geo-referenced data by using points, lines, polygons and circles. These imaginary surfaces are further digitally mapping and overlapping to the geo-referenced stereo imagery in the vicinity of the airport. Any terrain, building, antenna, or other object, which is higher than its relative restrictive height, will penetrate its relative transparent imaginary surface and be visually identified.

By applying the analytical and geometric method to the specific runway and its intended operations type, the dimensions of imaginary surfaces will be calculated, the imaginary surfaces along 3D coordinates will be constructed, and their critical points will be identified. Each critical point represents an endpoint or cross section between lines, circles and inclined planes according to the imaginary surface affecting it. The critical points are defined by their $x-y-z$ attributes in $3 \mathrm{D}$ coordinates with the origin point in the center of runway. Fig. 2 shows the imaginary surfaces and their critical points projecting into 3D coordinates. Based on the critical points which depict the required dimensions for the regulations, each imaginary surface is constructed by applying intensive calculation [5].

Taking the example of the approach surface, it is composed of two inclined trapezoids, one elevated horizontal surface and one inclined arc. Based on the given dimensions of the Category E precision instrument runway, the baselines of inclined trapezoids are calculated first and shown as follows:

$\overline{C C^{\prime}}=1,000 \mathrm{ft}$ in width, $200 \mathrm{ft}$ in distance, $0 \mathrm{ft}$ in height;

$\overline{j j}=3,250 \mathrm{ft}$ in width, $7,700 \mathrm{ft}$ in distance, $150 \mathrm{ft}$ in height;

$\overline{q q^{\prime}}=4,600 \mathrm{ft}$ in width, $12,200 \mathrm{ft}$ in distance, $250 \mathrm{ft}$ in height;

$\overline{u u^{\prime}}=16,000 \mathrm{ft}$ in width, $50,200 \mathrm{ft}$ in distance, $1,200 \mathrm{ft}$ in height.

$E L$ (elevation) and $H L$ (half runway length) have to be added into for specific runway.

Since the calculation result found that allowable height of segment between $\overline{j j^{\prime}}$ and $\overline{q q^{\prime}}$ is higher than horizontal and conical surfaces in the location, the elevated surface and inclined arc are constructed for approach surface. In order to generalize the applicability of the model, the designed imaginary surfaces are independent of site-specific inputs of runway length, width, location, orientation and elevation. Thus, the critical points are digitized as that the attribute $x$ is equal to extended width, the attribute $y$ is equal to distance plus half of runway length and attribute $z$ is equal to maximum allowable height plus runway elevation. In here, denotations are done as $x-y-z$ attributes, $c(500,200+H L, 0+E L), j '(-1,625,7700+$ $H L, 150+E L), q(2,300,12,200+H L, 250+E L), u^{\prime}$ $(-8,000,50,200+H L, 1,200+E L)$.

Based on the same analytical and geometric method, the critical points of every imaginary surface are solved 


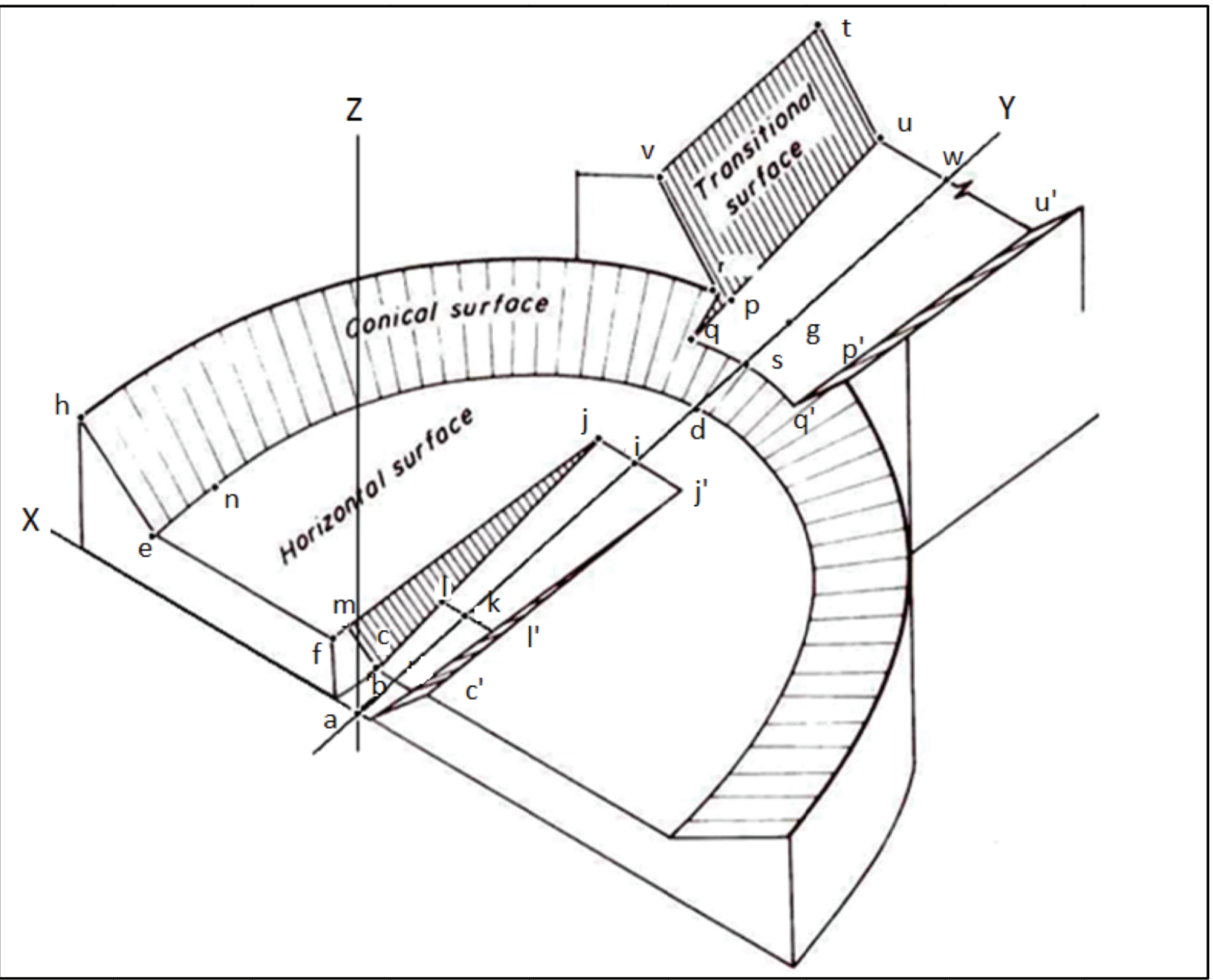

Fig. 2 Imaginary surfaces \& critical points [5].

gradually. The $x-y$ attributes are firstly specified by manually-entered flat surface coordinates. This 2D mock-up model in flat surface coordinates is rotated into its runway orientation and projected into UTM coordinate system. After converting, the geo-referenced 2D mock-up model is transformed into WGS84 system, which is the reference coordinate system used by the GPS. The attribute $z$ is equal to vertical height plus runway elevation. By using the $x-y-z$ attributes of critical points, the imaginary surfaces, which graphically represent the FAR Part 77 regulations, are generated. They display as different color of straight lines, curves and inclined planes in 3D and are overlapping on the geo-referenced stereo imagery in the vicinity of the airport. The imaginary surfaces represent the restrictive (i.e., maximum allowable) heights and locations of the potential obstacles for specific runway and its operations type. With the support of the DTM (digital terrain model), if the height of terrain is higher than maximum allowable height in its location, the extra obstruction that penetrates the $3 \mathrm{D}$ transparent imaginary surfaces will be visually identified. The existing manmade obstructions such as building, tower, antenna or crane will be also identified visually, but not for natural growth trees.

Fig. 3 shows the GIS Obstruction Model for precision instrument runway of Taipei Songshan Airport (IATA airport code: TSA; ICAO airport code: RCSS). Combined with existing obstruction database, 


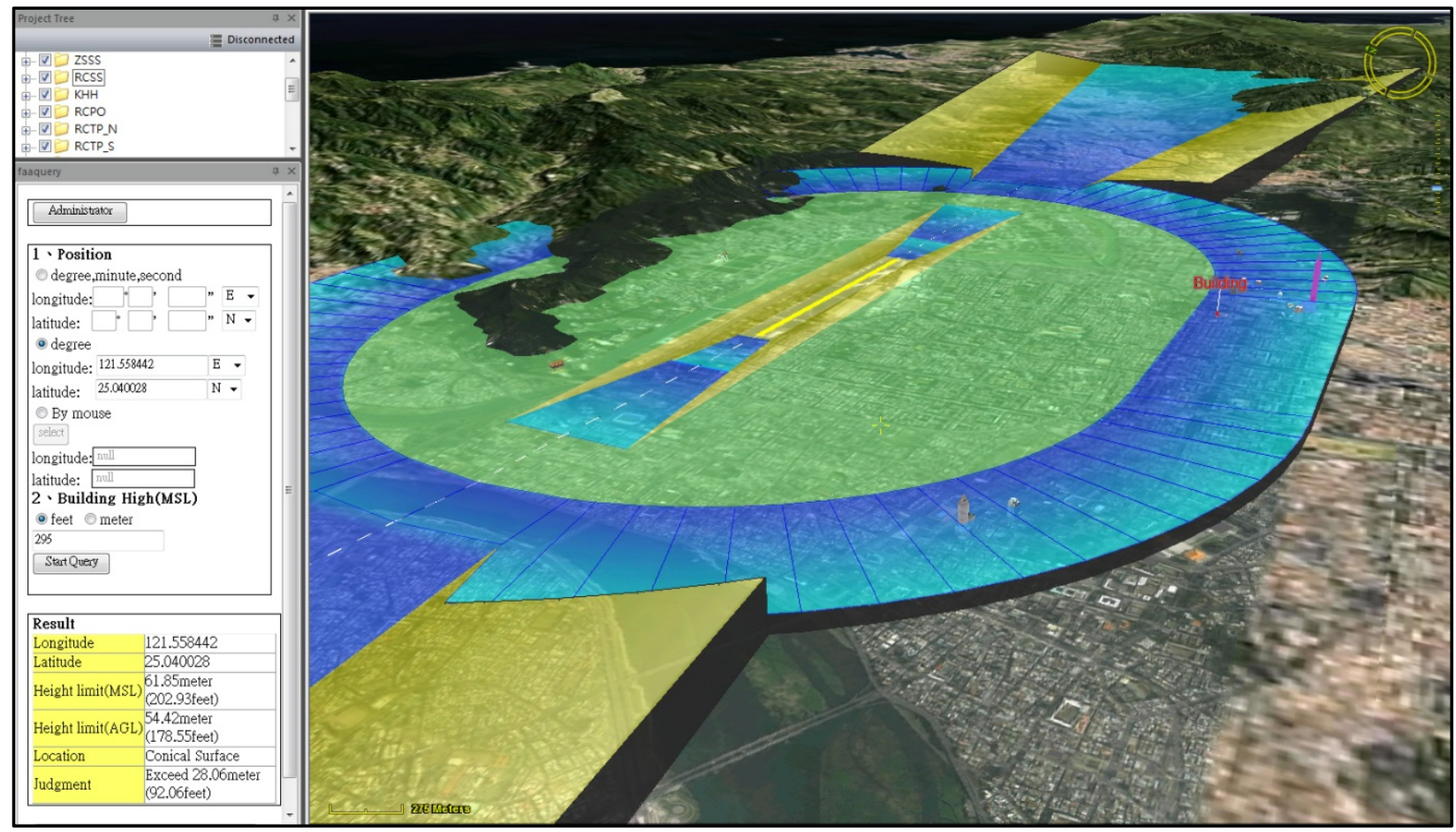

Fig. 3 OIS model of TSA Airport.

the terrain or building obstructions that penetrate the 3D transparent imaginary surfaces are visually identified and comparing with maximum allowable height. In order to help the user to find the maximum allowable height of the specific location in the vicinity of airport, an inquiry function is designed, as shown on the pull-down menu on the left side (Fig .3). That is allowed to input the exact location with latitude-longitude coordinates of specific location and inquiring height. It then promptly yields the restrictive (i.e., maximum allowable) height of this location with both figures and visual symbols. Several design features can be described as follows:

- A single, web-based database system for validated data in support of airport design and construction programs is used;

- The model is designed to be sufficiently general for application to different runways and allows site-specific inputs for runway length, width, location, orientation and elevation, enabling the practitioners to quickly generate an obstruction model for various uses;

- A friendly interface support with which new obstruction objects can be identified and previous existing data can be reviewed for accuracy and validity;

- 3D visualization of the obstructions can be achieved (e.g., terrain, buildings or antennas);

- It owns an inquiry function that allows manual entry of latitude-longitude coordinates or mouse-click selection of the location of new proposed construction;

- Quick response to online inquiries of restrictive height with both figures and visual symbols in real-time stereo mode is designed for the model;

- The model is designed with an inherently quantitative nature. It generates obstruction identification surfaces precisely, calculates obstacles location accurately, and precisely computes the restrictive height of the obstacles in the location.

With all of these special design features, the GIS Obstruction Model achieves the objective of this paper mentioned. It represents an up-to-date obstruction model for an airport with one runway or independent parallel runways. The design of the obstruction model for airports with multiple crossing runways or closely-spaced parallel runways requires further efforts 
in the future. The proposed model should be useful to transportation infrastructure planers in determining the airport location and runway orientation, to building developers in determining the restrictive height of proposed buildings, to government officials in evaluating the proposed constructions, and to airfield safety managers in monitoring obstructions and safety separations.

\section{Numerical Examples and Comparison}

In order to verify the model's design features, the problem of the 101 Building obstruction to Taipei Songshan Airport is analyzed both with hand calculations and this proposed model. The Songshan Airport has one precision instrument runway whose length is $8,547 \mathrm{ft}$ and whose width is $197 \mathrm{ft}$. The runway orientation is $10 \sim 28$. The runway elevation AMSL (above mean sea level) is $18 \mathrm{ft}$. The Taipei 101 Building is located on a bearing of $184^{\circ}$ and distance of $12,467 \mathrm{ft}$ off the end centerline of Runway 28. For the precision instrument runway, the dimensional standard of Category $\mathrm{E}$ is applied. Based on runway information, the size and direction of each surface have to be calculated and drawn in a relatively small scale map. Then, based on the building location, the surfaces affecting the building will be determined. The building in the example problem is located under the conical surface and horizontal surface. The maximum allowable height of the building can be solved as follows:

$$
\begin{gathered}
h=e_{r}-e_{b}+h_{h}+h_{c} \\
=e_{r}-e_{b}+h_{h}+\left(d_{b}-d_{h}\right) \times r_{c} \\
=18-0+150+(12,467-10,000) \times \frac{1}{20} \\
=291 \mathrm{ft}
\end{gathered}
$$

where:

$h=$ restrictive (i.e., maximum allowable) height of the building location AGL (above ground level);

$e_{r}=$ runway elevation AMSL;

$e_{b}=$ elevation difference of building location; $h_{h}=$ restrictive height of horizontal surface;

$h_{c}=$ restrictive height of selected location in conical surface;

$d_{b}=$ building distance to the end centerline of Runway 28;

$d_{h}=$ arc distance of horizontal surface;

$r_{c}=$ slope ratio of conical surface.

Compared to hand calculation, using the GIS Obstruction Model to determine the restrictive height of the building is much easier and accurate. After opening the web page of the Songshan Airport GIS Obstruction Model, the imaginary surfaces over-lapping with digital terrain (Fig. 3) are shown. By inputting the latitude and longitude of the building location (or simply clicking to select the location) and inquiring height of the proposed building, the model determines in real time that the restrictive height is $291.33 \mathrm{ft}$ and visually indicates the building under specific surface on which the building is located. The proposed GIS Obstruction Model has been verified for accuracy in calculations and proved an up-to-date design.

The container terminals of Kaohsiung Seaport are located close to the approach surface of the Runway 09 of Kaohsiung International Airport (IATA airport code: $\mathrm{KHH}$; ICAO airport code: RCKH). Some types of container gantry cranes, such as super-post Panamax, reach $90 \mathrm{~m}(295 \mathrm{ft})$ in total height. When their booms are lifted in the air to clear the ships for navigation, they are higher than the maximum allowable height and penetrate the OISs. Using the proposed model to solve this problem is very simple, comparing to measure and calculate every location of height of hundreds of cranes. The nearest allowable location of $295 \mathrm{ft}$ crane is the distance of $12,950 \mathrm{ft}$ from the center of runway. From GIS Obstruction Model of $\mathrm{KHH}$ Airport shown in Fig. 4, it can be visually identified the OIS penetration of hazardous obstruction if any crane is located in horizontal surface, inner three-quarters of conical surface or inner one-third of approach surface. 


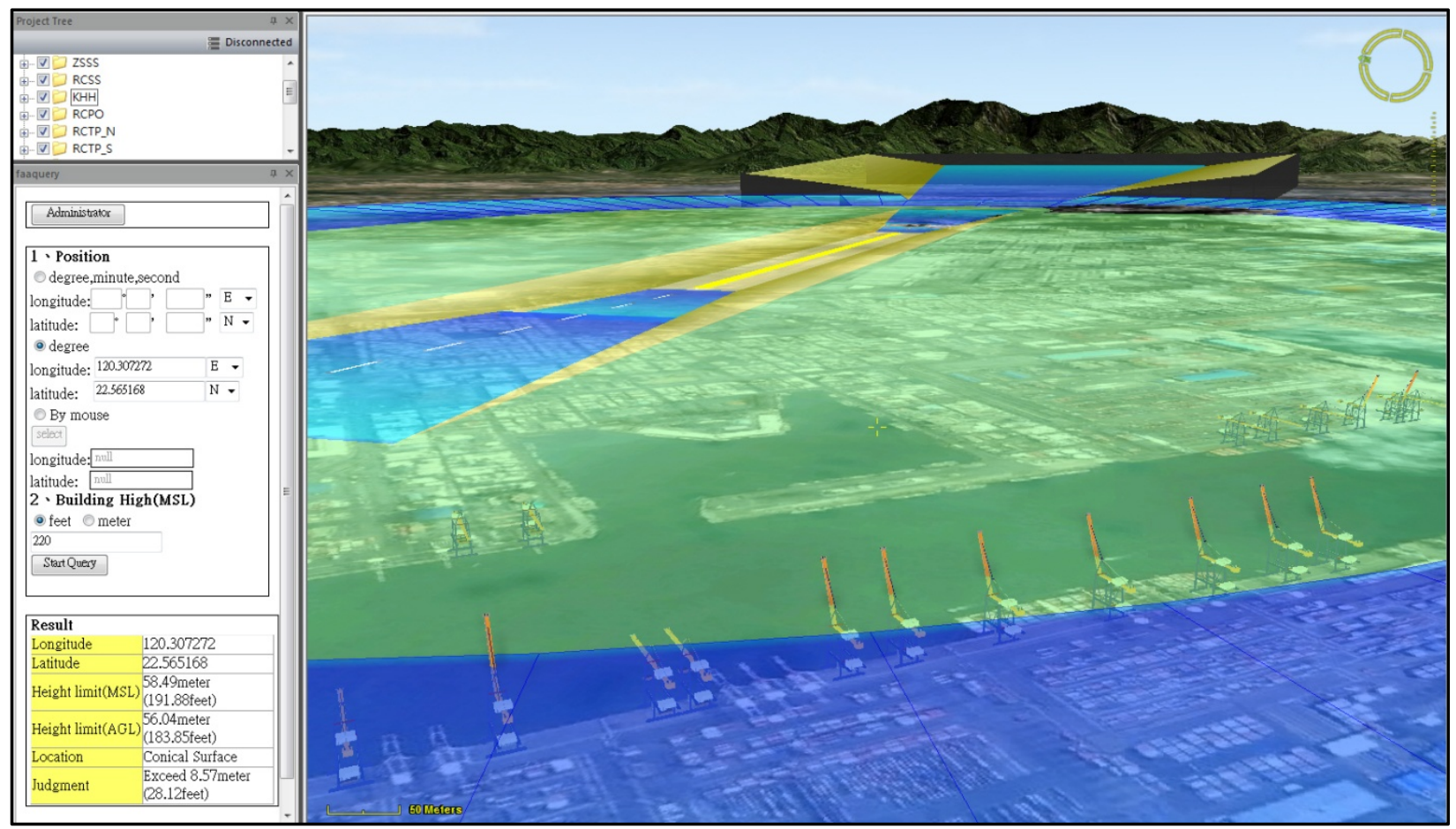

Fig. 4 OIS model of KHH Airport.

\section{Conclusions}

The OIS evaluation is often used for new airfield site selection and runway orientation determination. More importantly, the OIS evaluation is an airport's safeguard against flawed urban development, especially for airports near rapidly expanding cities. Without accurate and handy tools, hand-calculation errors on the restrictive height/area of the OIS could occur and cause adverse impacts to airport safety. Applying the GIS features of geospatial data and 3D formats, this paper develops the GIS Obstruction Model which is capable of calculating restrictive height accurately and creating visual imagery of penetrating obstructions. The special features of the model include web-based design, adaptability to any airport, friendly multiple inquiry methods, accurate calculation, quick response to online inquiring, and response with 3D visual reality. The proposed GIS Obstruction Model presents an up-to-date design for an airport with one runway or multiple distant runways. An airport cost minimization model, which combines airfield land use and multiple runway orientation optimizations [11], is the long-term goal for airport design. The proposed model in this paper is one of the essential modules for this. Even the proposed GIS Obstruction Model itself, it has been demonstrated as an useful tool for airport design, transportation infrastructure planning, urban development and airfield safety management.

\section{References}

[1] FAA (Federal Aviation Administration). 1993. FAR Part 77: Objects Affecting Navigable Airspace. Washington, D.C.: FAA.

[2] TRB (Transportation Research Board). 2010. Understanding Airspace, Objects, and Their Effects on Airports. ACRP (Airport Cooperative Research Program) report 38, 8-40.

[3] Chang, S. W., and Chin, Y. R. 2010. "Comparison of Obstacle Restriction and Analysis of Safety Airspace between RCSS Airport and FAA OIS." In 2010 Proceeding of Aeronautical \& Astronautical Society, 217-23.

[4] Litsheim, S., and Xiao, X. 2009. "Airport Obstacle Surfaces." Journal of Advanced Transportation 43: 347-66.

[5] Horonjeff, R., and McKelvey, F. X. 1994. Planning and Design of Airport. 4th ed. New York: McGraw-Hill.

[6] Vidal, S., Graham, D., and Steele, J. 2004. "FAA Airport 
Surveying-GIS Program: The Presentation to Airport GIS Committee." National Geodetic Survey. Accessed March 30, 2009. http://www.ngs.noaa.gov/AERO/eAOC/p1635. pdf.

[7] Panayotov, A., Georgiev, I. K. 2008. "Modelling of the Airport Navigation Apace Obstructions." Presented at 3rd International Conference from Scientific Computing to Computational Engineering, Athens, Greece.

[8] Panayotov, A., Georgiev I., and Georgiev, I. 2012. "A Practical Approach for Airport Spatial Modeling." In Proceedings of the 13th International Conference on Computer Systems and Technologies,
321-8.

[9] Ulubay, A., and Altan, M. O. 2002. "A Different Approach to the Spatial Data Integration." Presented at 2002 Symposium on Geospatial Theory, Processing and Applications, Ottawa, Canada.

[10] ICAO (International Civil Aviation Organization). 2004. Aerodrome Design and Operations. Annex 14 to the Convention on International Civil Aviation. Quebec: ICAO.

[11] Chang, S. W. 2015. "Crosswind-Based Optimization of Multiple Runway Orientations." Journal of Advanced Transportation 49: 1-9. 